\title{
AVALIAÇ̃̃O NUMÉRICA E EXPERIMENTAL DA INFLUÊNCIA DE CONDIÇÕES OPERACIONAIS NA CARGA DE SÓLIDOS EM TAMBORES ROTATÓRIOS COM SUSPENSORES
}

\author{
S. M. NASCIMENTO ${ }^{1 *}$, L. F. G. de ÁVILA ${ }^{1}$, F.P. de LIMA ${ }^{1}$, M. A. S. BARROZO ${ }^{1}$, C. \\ R. DUARTE ${ }^{1}$ \\ ${ }^{1}$ Universidade Federal de Uberlândia, Faculdade de Engenharia Química \\ *e-mail: suellenmendonca01@gmail.com
}

\begin{abstract}
RESUMO
Os secadores rotatórios são equipamentos amplamente utilizados nos mais diversos tipos de indústrias. Apesar de ser objeto de numerosos estudos, ainda existe uma deficiência no estudo do comportamento dinâmico das partículas nestes equipamentos, sendo atualmente o projeto destes secadores baseados em resultados empíricos e em scale-up de plantas piloto. Este trabalho tem como objetivo estudar o efeito das condições operacionais na dinâmica do material particulado em tambores rotatórios. O modelo Euleriano Granular Multifásico foi adotado para predizer a carga de sólidos nos suspensores e os resultados foram comparados com o trabalho experimental usando uma metodologia criada especificamente para este estudo. Foram analisadas as influências do diâmetro da partícula, propriedades do material, velocidade de rotação e carregamento do tambor na carga de sólidos nos suspensores do tambor rotatório. Os resultados do presente trabalho mostraram que a abordagem Euleriana foi capaz de predizer o comportamento do material particulado no equipamento para diferentes materiais operando em diferentes condições operacionais. Apesar dos desvios do modelo em relação aos resultados experimentais, a abordagem Euleriana permitiu o desenvolvimento de um modelo mais generalizado quando comparado com outras modelagens, com um custo computacional relativamente baixo.
\end{abstract}

\section{INTRODUÇÃO}

Os secadores rotatórios são utilizados em diversos tipos de indústrias. Apesar de serem os tipos de secadores mais utilizados, eles representam um custo significativo para muitas indústrias e sua eficiência térmica está diretamente relacionada como projeto e operação adequados do equipamento (LEE, 2008).
Diversos estudos possuem como foco a caracterização da dinâmica das partículas em tambores rotatórios, mas o projeto desses equipamentos é complexo e ainda ditado por fatores empíricos. Desta maneira, são necessários estudos teóricos para que sejam compreendidos os fundamentos da dinâmica de material particulado nesses secadores.

De acordo com Lee (2008), idealmente, um secador rotatório deve ser operado de forma que a máxima quantidade de sólido 
esteja sendo descarregada através da cortina de ar em cada ponto.

De acordo com Ajayi e Sheehan (2012), o tambor pode ser classificado a depender da posição em que ocorre a primeira descarga no equipamento. Se a primeira descarga ocorre para uma posição angular acima de $0^{\circ}$ (posição em que passa a linha horizontal que divide o tambor ao meio), o secador estará subcarregado. Já quando a primeira descarga ocorre em regiões abaixo de $0^{\circ}$, o equipamento está sobrecarregado e o excesso de material sofre movimento de rolamento no fundo tambor. Desta maneira, um secador estará operando em seu carregamento ideal quando a primeira descarga ocorre precisamente na posição $0^{\circ}$.

O dimensionamento e a simulação de secadores rotatórios são grandes desafios, visto que a secagem envolve além transferências de calor e massa, uma dinâmica complexa do material particulado. Para isto, no estudo fluidodinâmico geralmente são utilizados tambores rotatórios com suspensores, mas sem a presença do ar de secagem, que facilitam o entendimento do movimento das partículas.

O CFD é uma ferramenta bastante útil na descrição de escoamentos, e aliada com os dados experimentais que validam os modelos, pode fornecer resultados satisfatórios em diversos processos.

As duas abordagens comumente utilizadas para a simulação da dinâmica de tambores rotatórios são a Euler-Euler e a de Lagrange (SANTOS et al., 2013). A abordagem Lagrangeana é baseada nas forças de interação partícula-partícula, enquanto que na abordagem Euler-Euler, as fases são tratadas matematicamente como contínuas e interpenetrantes.

A abordagem Lagrangeana, usada pelo DEM (Discrete Element Method), já tem sido utilizada para a simulação de tambores rotatórios com suspensores (GENG et al., 2013; SILVÉRIO et al., 2014). Porém, o
DEM possui um problema crucial, o custo computacional é diretamente proporcional ao número de partículas do processo e há a necessidade de um grande número de parâmetros para descrever o processo. $\mathrm{Na}$ abordagem Euler-Euler não é possível descrever uma partícula individualmente, porém o custo computacional desta abordagem é bem menor.

Apesar da abordagem Euler-Euler já ter sido utilizada para estudar o comportamento de uma cortina de partículas caindo através de uma corrente horizontal de gás (AJAYI e SHEEHAN, 2012; WARDJIMAN et al., 2008), não existem trabalhos que a utilizam a abordagem para descrever o comportamento das partículas em um tambor rotatório com suspensores.

O objetivo do presente trabalho, portanto, foi analisar o efeito de condições operacionais na dinâmica de sólidos em tambor rotatório com suspensores, através de resultados experimentais e realizar simulações via $C F D$, utilizando a abordagem Euler-Euler.

\section{MATERIAIS E MÉTODOS}

\subsection{Aparato Experimental}

O aparato experimental, mostrado na Figura 1, consiste de um tambor cilíndrico com 10,8 cm de diâmetro e $50 \mathrm{~cm}$ de comprimento $(\mathrm{L} / \mathrm{D}=5)$, fabricado em aço inoxidável. $\mathrm{Na}$ parte interna do tambor existem seis suspensores igualmente espaçados com três segmentos, de comprimento de 10,4 e $4 \mathrm{~mm}$, visto que recomenda-se que a profundidade do suspensor esteja entre $\mathrm{D} / 8$ e D/12. $\mathrm{Na}$ extremidade frontal do tambor, foi colocado um visor de vidro temperado que permite fotografar a posição angular do suspensor e na outra extremidade foi montado um sistema em acrílico para coleta de partículas. A velocidade de rotação do tambor era medida utilizando um tacômetro digital. 
Figura 1 - Visão frontal do equipamento utilizado no trabalho experimental.

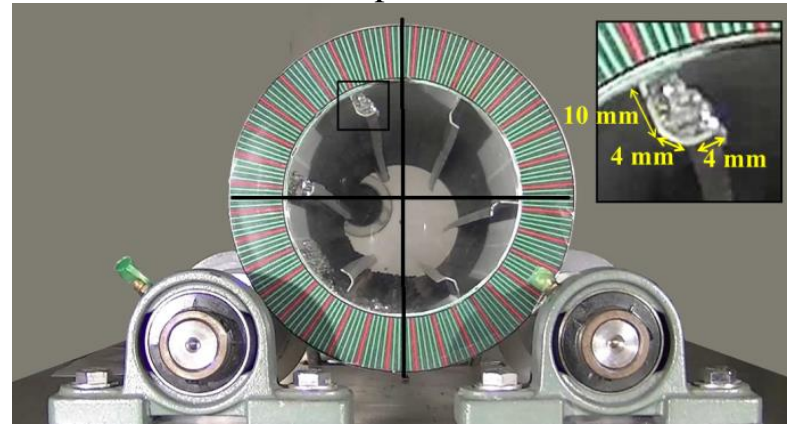

As partículas usadas foram esferas de vidro com diâmetro médio de peneira de 1,09 , 1,84 e $2,56 \mathrm{~mm}$ e densidade bulk de 2.455 $\mathrm{kg} / \mathrm{m}^{3}$. Para as partículas de diâmetro médio de $2,56 \mathrm{~mm}$, a porosidade medida do leito consolidado foi de 0,368 . As velocidades de rotação do tambor utilizadas foram 1,5, $3 \mathrm{e}$ $4,5 \mathrm{rpm}$. Além das esferas de vidro, foram utilizados fertilizantes super fosfato simples granulado (SSP) com um diâmetro médio de 2,56 mm e uma densidade bulk, medida com glicerina, de $2.090 \mathrm{~kg} / \mathrm{m}^{3}$. Para o fertilizante, a porosidade medida para o leito empacotado foi de 0,515 e a velocidade de rotação foi de 3 rpm. A densidade dos materiais foi medida através do método de picnometria e a porosidade do leito usando uma proveta de 50 $\mathrm{mL}$.

A posição angular da ponta do suspensor foi medida através de análise de imagens. Uma câmera em um tripé foi posicionada na horizontal, e era medido o ângulo entre a linha formada pela ponta do suspensor e a origem e o eixo horizontal, utilizando software Image $J^{\circledR}$. Quando um suspensor atingia uma determinada posição angular, a rotação era interrompida e a posição angular medida usando o Image $^{\circledR}$. Posteriormente, as partículas eram coletadas e pesadas. Com este procedimento, a carga de sólidos era medida como uma função da posição angular.

Outro aparato experimental foi usado para medir o ângulo de fricção interna partícula-partícula, o qual será denotado por $\beta$. Algumas partículas são aderidas à superfície de um plano inclinado e outras colocadas livremente sobre essas. Quando as partículas livres começam a rolar no plano, este é o ângulo de fricção interna partículapartícula. Os valores medidos foram $28^{\circ} \pm 0,6^{\circ}$ para as esferas de vidro de $2,56 \mathrm{~mm}$ de diâmetro e $41^{\circ} \pm 0,8$ para o fertilizante SSP granulado de mesmo diâmetro.

\subsection{Simulação CFD}

2.2.1 Equações de conservação de massa e momento e modelo de arraste

Foi adotado o modelo Euleriano Granular Multifásico acoplado à Teoria Cinética do Escoamento Granular, desenvolvida por Lun et al. (1984). A Tabela 1 descreve as equações fundamentais $\mathrm{e}$ constitutivas utilizadas no modelo.

Os termos $v_{s}, \vec{v}_{f}, V_{f}, \alpha_{S}, \alpha_{f}, C_{D}, R e_{r}, \phi_{f s}, k_{\theta S}$ , $K_{s f}, g_{0, s s}, \mathrm{e}_{s s}, \gamma_{\theta S}$ são o vetor velocidade da fase sólida, o vetor velocidade da fase fluida, o volume da fase fluida, a fração volumétrica da fase sólida e da fase fluida, o coeficiente de arraste, número de Reynolds relativo, a troca de energia entre as fases sólida e fluida, o coeficiente de difusão de energia, a troca de momento sólido-fluido, a função de distribuição radial, o coeficiente de restituição da fase sólida e a dissipação de energia colisional, respectivamente.

Quando a concentração de partículas é alta, a viscosidade friccional $\left(\mu_{s, \text { fric }}\right)$ deve ser somada à viscosidade colisional $\left(\mu_{s, c o l}\right)$ e à cinética $\left(\mu_{s, k i n}\right)$ para estimar a viscosidade da fase sólida.Para o cálculo da viscosidade friccional foi usado o modelo de Schaeffer (1987) apresentado pela Equação 16.

$$
\mu_{\mathrm{s}, \mathrm{fr}}=\frac{P_{S} \operatorname{sen}(\beta)}{2 \sqrt{I_{2 D}}}
$$


Tabela 1- Principais equações fundamentais e constitutivas do modelo.

\begin{tabular}{cc}
\hline & Equações fundamentais \\
\hline $\begin{array}{c}\text { Conservação de massa da } \\
\text { fase fluida }\end{array}$ & $\frac{\partial}{\partial t}\left(\alpha_{f}\right)+\nabla \cdot\left(\alpha_{f} \vec{v}_{f}\right)=0$ \\
$\begin{array}{c}\text { Conservação de massa da } \\
\text { fase sólida } \\
\text { Conservação de }\end{array}$ & $\frac{\partial}{\partial t}\left(\alpha_{s}\right)+\nabla \cdot\left(\alpha_{s} \vec{v}_{s}\right)=0$ \\
$\begin{array}{c}\text { momentum da fase fluida } \\
\text { Conservação de }\end{array}$ & $\frac{\partial}{\partial t}\left(\alpha_{f} \rho_{f} \vec{v}_{f}\right)+\nabla \cdot\left(\alpha_{f} \rho_{f} \vec{v}_{f} \vec{v}_{f}\right)=-\alpha_{f} \nabla p+\nabla \cdot \overline{\bar{\tau}}_{f}+\alpha_{f} \rho_{f} \vec{g}+\left(K_{s f}\left(\vec{v}_{s}-\vec{v}_{f}\right)\right)$ \\
$\begin{array}{c}\text { momentum da fase sólida } \\
\text { Equação de transporte da } \\
\text { temperatura granular }\end{array}$ & $\frac{\partial}{\partial t}\left(\alpha_{s} \rho_{s} \vec{v}_{s}\right)+\nabla \cdot\left(\alpha_{s} \rho_{s} \vec{v}_{s} \vec{v}_{s}\right)=-\alpha_{s} \nabla p-\nabla p_{s}+\nabla \cdot \overline{\bar{\tau}}_{s}+\alpha_{s} \rho_{s} \vec{g}+\left(K_{s f}\left(\vec{v}_{f}-\vec{v}_{s}\right)\right)$ \\
\hline
\end{tabular}

Tensor de cisalhamento

da fase sólida

\section{Equações constitutivas}

$$
\begin{gathered}
\overline{\bar{\tau}}_{s}=\alpha_{s} \mu_{s}\left(\nabla \vec{v}_{s}+\nabla \vec{v}_{s}^{T}\right)+\alpha_{s}\left(\lambda_{s}-\frac{2}{3} \mu_{s}\right) \nabla \vec{v}_{s} \bar{I} \\
\overline{\overline{\tau_{f}}}=\mu_{f}\left[\nabla \overrightarrow{v_{f}}+\left(\nabla \overrightarrow{v_{f}}\right)^{T}\right]-\frac{2}{3} \mu_{f}\left(\nabla \cdot \overrightarrow{v_{f}}\right) \overline{\bar{I}}
\end{gathered}
$$

Tensor de cisalhamento da fase fluida

Coeficiente de transferência da

quantidade de movimento

$$
K_{S f}= \begin{cases}K_{s f_{-} \text {Wen_Yu}} & \alpha_{f}>0,80 \\ K_{s f_{-} \text {Ergun }} & \alpha_{f} \leq 0,80\end{cases}
$$

$$
K_{s f_{-} W e n_{-} Y u}=\frac{3}{4} C_{D} \frac{\alpha_{s} \alpha_{f} \rho_{f}\left|\vec{v}_{s}-\vec{v}_{f}\right|}{d_{s}} \alpha_{f}^{-2,65}
$$

$$
\begin{gathered}
K_{s f_{-} \text {Ergun }}=150 \frac{\alpha_{s}\left(1-\alpha_{f}\right) \mu_{f}}{\alpha_{f} d_{s}^{2}}+1,75 \frac{\rho_{f} \alpha_{s}\left|\vec{v}_{s}-\vec{v}_{f}\right|}{d_{s}} \\
C_{D}= \begin{cases}\frac{24}{\left(\operatorname{Re}_{r} \alpha_{f}\right)}\left[1+0,15\left(\operatorname{Re}_{r} \alpha_{f}\right)^{0,687}\right], & \operatorname{Re}_{r} \leq 1000 \\
0,44, & \operatorname{Re}_{r}>1000\end{cases}
\end{gathered}
$$

$$
\operatorname{Re}_{r}=\frac{\rho_{f}\left|\vec{v}_{f}-\vec{v}_{s}\right| d_{s}}{\mu_{f}}
$$

Pressão de sólidos

Função de distribuição radial

Coeficiente de difusão de energia granular

Dissipação de energia colisional

$$
P_{s}=\alpha_{s} \rho_{s} \theta_{s}+2 \rho_{s}\left(1+e_{s s}\right) \alpha_{s}^{2} g_{0, s s} \theta_{s}
$$

$$
g_{0, s s}=\left[1-\left(\frac{\alpha_{s}}{\alpha_{s, \max }}\right)^{1 / 3}\right]^{-1}
$$

$$
k_{\theta s}=\frac{150 \rho_{S} d_{s} \sqrt{\theta_{s} \pi}}{384\left(1+e_{s s}\right) g_{0, s s}}\left[1+\frac{6}{5}\left(1+e_{s s}\right) \alpha_{s} g_{0, s s}\right]^{2}+2 \alpha_{S}^{2} \rho_{S} d_{s} g_{0, s s}\left(1+e_{s s}\right) \sqrt{\frac{\theta_{s}}{\pi}}
$$

$$
\gamma_{\theta s}=3\left(1-e_{s s}{ }^{2}\right) \alpha_{s}^{2} \rho_{s} g_{0, s s} \theta_{s}\left(\frac{4}{d_{s}} \sqrt{\frac{\theta_{s}}{\pi}}-\nabla \cdot \overrightarrow{v_{s}}\right)
$$


A viscosidade colisional foi estimada de acordo com a equação 17:

$$
\mu_{S, c o l}=\frac{4}{5} \alpha_{S} \rho_{S} d_{S} g_{0, S S}\left(1+e_{S S}\right)\left(\frac{\theta_{S}}{\pi}\right)^{1 / 2} \alpha_{S}
$$

A viscosidade cinética foi estimada com o modelo de Gidaspow (1994):

$$
\mu_{S, k i n}=\frac{10 \rho_{S} d_{S} \sqrt{\theta_{S} \pi}}{96 \alpha_{S}\left(1+e_{S S}\right) g_{0, S S}}\left[1+\frac{4}{5} g_{0, S S} \alpha_{S}\left(1+e_{S S}\right)\right]^{2} \alpha_{S}
$$

\subsubsection{Condições de simulação}

As simulações foram realizadas no software FLUENT ${ }^{\circledR}$ 14.0. Foi utilizada uma malha tridimensional (3D) e o domínio de cálculo foi discretizado em 248.500 células. $\mathrm{O}$ método de discretização foi através de volumes finitos e o acoplamento pressãovelocidade foi feito utilizando o algoritmo SIMPLE. Uma condição de malha móvel foi utilizada.

Para a interpolação do centro da célula para as fronteiras foi utilizado o método de interpolação First-Order-Upwind-method. O coeficiente de restituição $\left(e_{s s}\right)$ utilizado foi de 0,9 , o time step de $1 \times 10^{-4} \mathrm{~s}$ e cada simulação foi conduzida para um período de $60 \mathrm{~s}$, tempo em que não havia variação de holdup nos suspensores entre duas voltas do tambor. $\mathrm{O}$ erro relativo entre duas iterações sucessivas foi de $1 \times 10^{-3}$ para cada variável analisada. A velocidade de rotação utilizada foi $3 \mathrm{rpm} \mathrm{e}$ foram feitas simulações para esferas de vidro e fertilizante SSP com 2,56 mm de diâmetro.

\subsubsection{Estimativa de holdup}

Como o software FLUENT $^{\circledR}$ não fornece o holdup de forma direta foi necessário o desenvolvimento de uma metodologia para estimá-lo. Os perfis de escoamento obtidos nas simulações usando o software FLUENT $^{\circledR}$ apresentam uma escala de cores, como mostrada na Figura 2.
Figura 2 - Variação da fração de sólidos na seção transversal do tambor simulado e experimental.

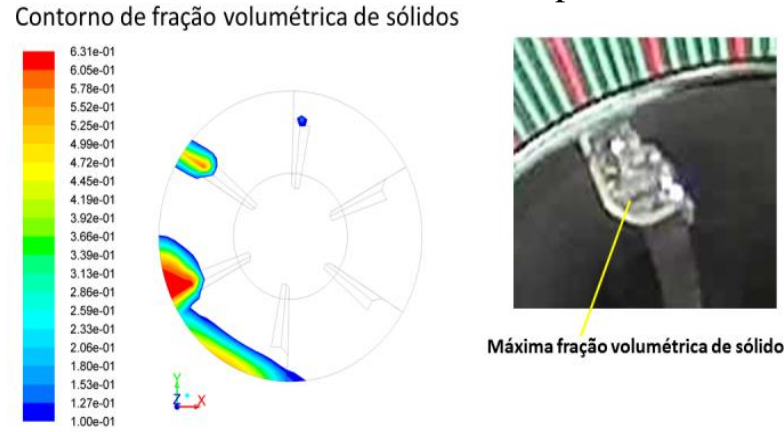

Foi observado que no trabalho experimental não havia uma faixa de concentração volumétrica de partículas como na simulação. Observou-se que esta escala de cores foi um resultado da interpolação na resolução numérica. Numericamente, a transição da máxima concentração volumétrica de sólidos para a ausência de partículas não é brusca, como observado experimentalmente (Figura 2). A transição gradual entre as duas situações dá origem à variação da fração volumétrica de sólidos em diferentes regiões do suspensor.

Para o cálculo da carga de sólidos nos suspensores, na simulação, a massa de cada cor foi calculada com base na Equação 19.

$m_{i}=a_{i}\left(\frac{\alpha_{\mathrm{max}, \mathrm{i}}+\alpha_{\mathrm{min}, \mathrm{i}}}{2}\right) \rho_{S} L$

onde $m_{i}$ é a massa correspondente a cada cor, $a_{i}$ é a área ocupada pela cor, ${ }^{\alpha \max , \mathrm{i}}$ é a fração de sólidos máxima e $\alpha_{\text {min,i é a fração de }}$ sólidos mínima de cada cor e $L$ é o comprimento do tambor.

A carga de sólidos no suspensor $\left({ }^{h}\right)$, nas simulações é obtida pela soma das massas de cada cor (Equação 20).

$h=\sum_{i=1}^{20} m_{i}$ 


\section{RESULTADOS E DISCUSSÃO}

O carregamento ideal deve ser estimado para garantir a qualidade do produto final. No presente estudo, o holdup como uma função da posição angular foi medido para diferentes velocidades de rotação do tambor, cargas de sólidos no tambor, diâmetro de partícula e tipos de material.

A Figura3 mostra o perfil de descarga dos suspensores para esferas de vidro de 1.09 $\mathrm{mm}$ em três diferentes velocidades de rotação.

Figura 3-Massa de sólidos no suspensor para esferas de vidro de 1,09mm de diâmetro em três velocidades de rotação: (a) 1,5 rpm; (b) $3 \mathrm{rpm}$ e (c) 4,5 rpm, para diferentes carregamentos do tambor.

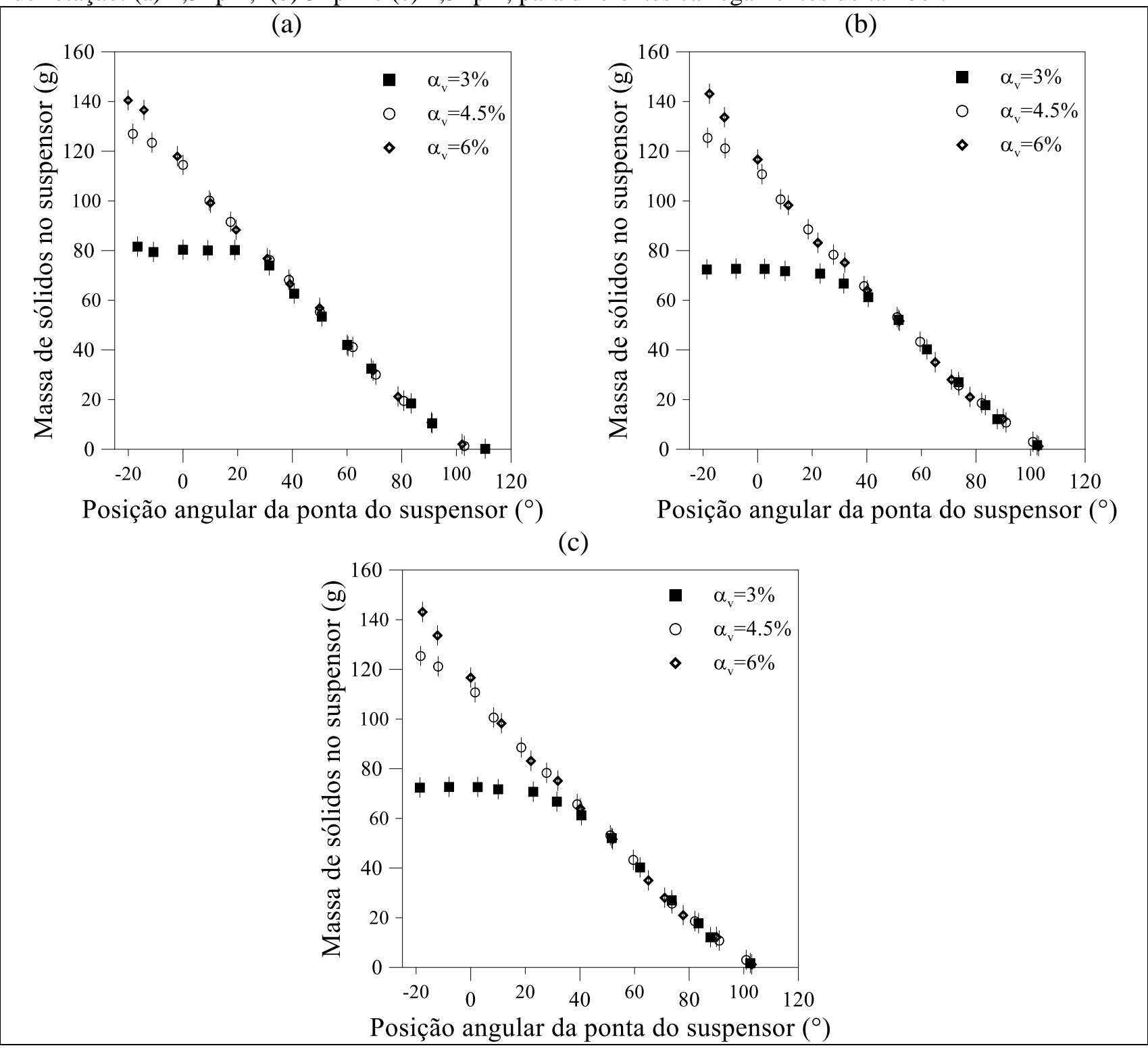

Observa-se que quando a fração de sólidos no tambor ocupa 3\% do tambor em fração volumétrica, o tambor está abaixo do carregamento ideal, em qualquer uma das velocidades, tendo em vista que a massa de sólidos só começa a descarregar em torno da 
posição angular de $40^{\circ}$. Para o caso em que a fração volumétrica das esferas ocupa $4,5 \%$ e $6 \%$, para o diâmetro de $1,09 \mathrm{~mm}$, o tambor está operando acima do seu carregamento ideal, visto que as partículas estão sendo descarregadas antes da posição $0^{\circ}$.

$\mathrm{Na}$ Figura 4, estão apresentados os perfis de descarga dos suspensores para esferas de vidro com diâmetro médio de 1,84 $\mathrm{mm}$, em três velocidades de rotação. Observa- se que para um grau de enchimento do tambor de 4,5\%, o tambor está com um carregamento ideal (início da descarga em $0^{\circ}$ ). Para um grau de enchimento de $3 \%$ o tambor está subcarregado e para $6 \%$ ele está sobrecarregado. Este resultado foi observado em todas as velocidades analisadas. Para as esferas de vidro de $2,56 \mathrm{~mm}$ de diâmetro médio, os resultados são os mesmos e o carregamento ideal se dá também em 4,5\%.

Figura 4 - Massa de sólidos no suspensor para esferas de vidro de 1,84mm de diâmetro em três velocidades de rotação: (a) $1,5 \mathrm{rpm}$; (b) $3 \mathrm{rpm}$ e (c) 4,5 rpm, para diferentes carregamentos do tambor.

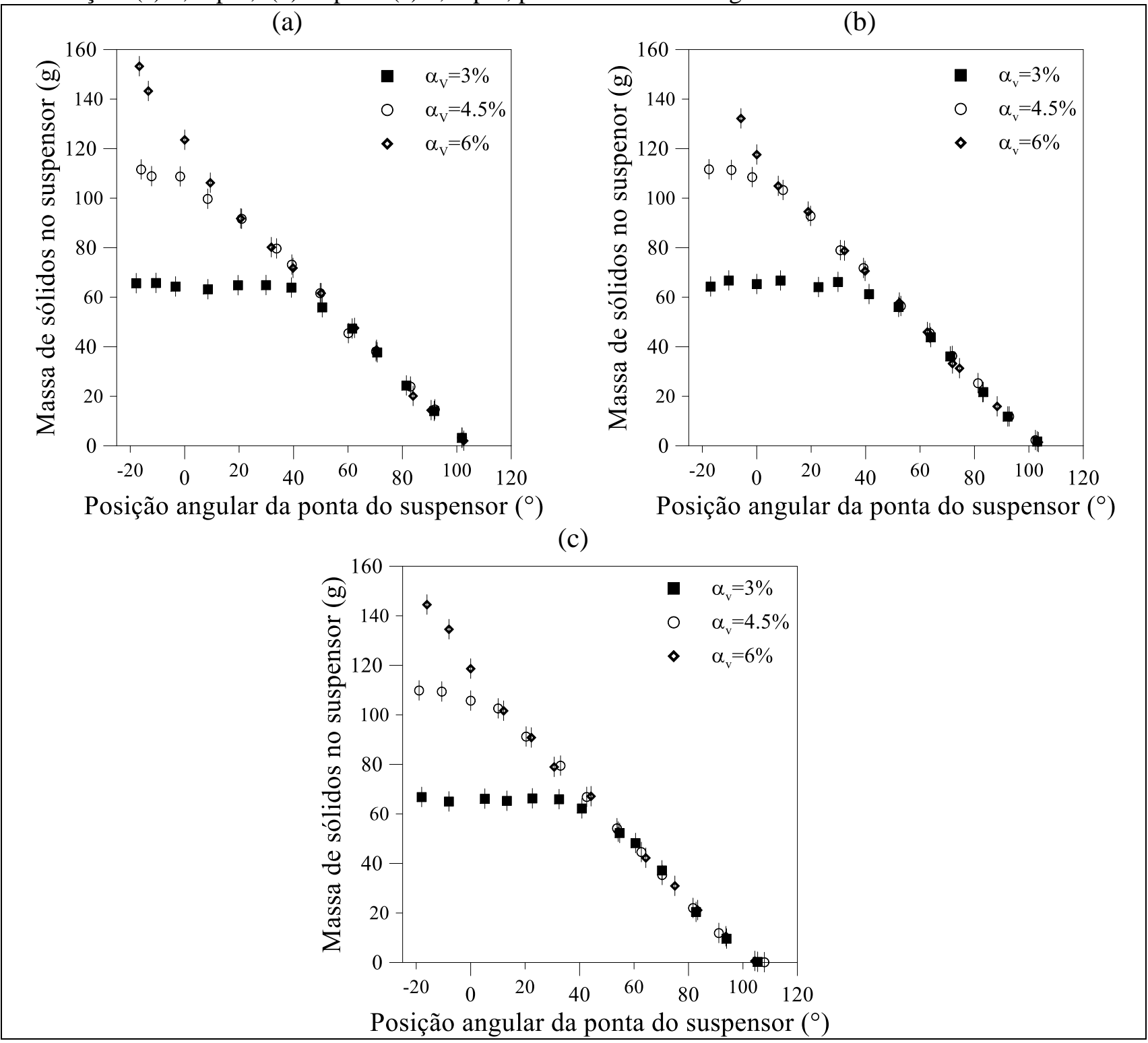


Como pode ser observado nas Figuras 3 e 4, o carregamento ideal não foi dependente da velocidade para as três variáveis analisadas, que geralmente são baixas em secadores rotatórios. No entanto, mostrou-se dependente do diâmetro da partícula. Assim, como as esferas de vidro de $1,09 \mathrm{~mm}$ escoam melhor do que as partículas com maiores diâmetros, o carregamento ideal para as esferas de $1,09 \mathrm{~mm}$ é ligeiramente mais baixo do que para as partículas com diâmetro médio de 1,84 e 2,56 mm.

$\mathrm{Na}$ figura 5, é apresentado o holdup de sólidos nos suspensores como uma função da posição angular para o fertilizante de $2,56 \mathrm{~mm}$ de diâmetro e uma velocidade de rotação do tambor de $3 \mathrm{rpm}$.

Figura 5- Massa de sólidos no suspensor para fertilizante SSP de $2,56 \mathrm{~mm}$ de diâmetro para diferentes carregamentos do tambor.

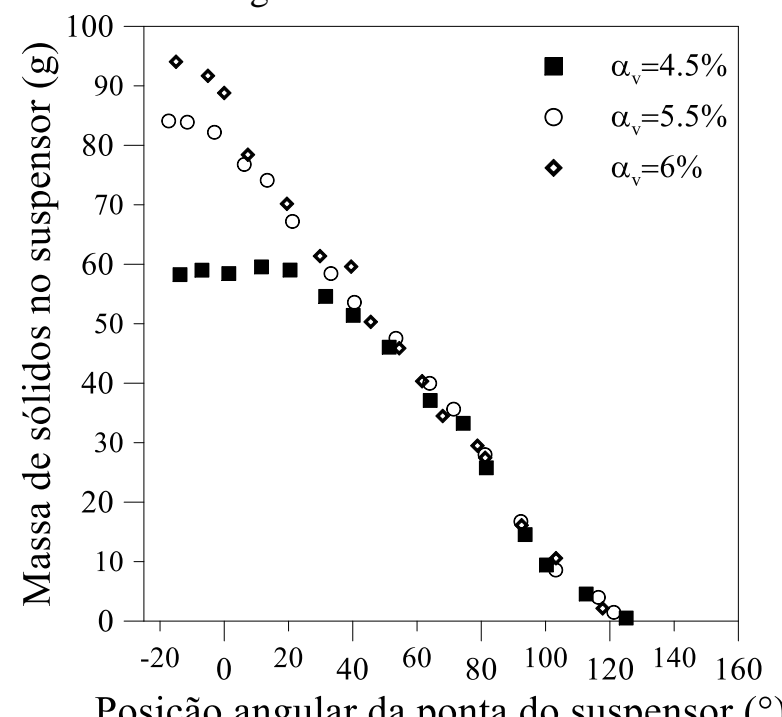

Observou-se que para esta partícula o carregamento ideal de se dá para um grau de enchimento de $5,5 \%$, maior que o observado para as esferas de vidro de mesmo diâmetro. O carregamento ideal é maior para o fertilizante do que para as esferas de vidro porque o fertilizante é mais coesivo. Os valores experimentais medidos para o ângulo de repouso estático foram $28^{\circ} \pm 0,6^{\circ}$ para as esferas de vidro de $2,56 \mathrm{~mm}$ de diâmetro e $41^{\circ} \pm 0,8^{\circ}$ para o fertilizante SSP de mesmo diâmetro, justificando assim a diferença encontrada nos valores de carregamento ideal para estes materiais.

Outro objetivo do trabalho foi investigar o modelo usado para determinar o holdup e comparar os resultados preditos por CFD e os dados experimentais. A Figura 6 mostra a variação do holdup de sólidos simulado por CFD e experimental em função da posição angular. Foi usado um grau de enchimento de $6 \%$ e esferas de vidro com um diâmetro médio de $2,56 \mathrm{~mm}$.

Figura 6 - Comparação entre massas de sólido experimental e simulado para esferas de vidro de $2,56 \mathrm{~mm}$ e carga de sólidos de $6 \%$.

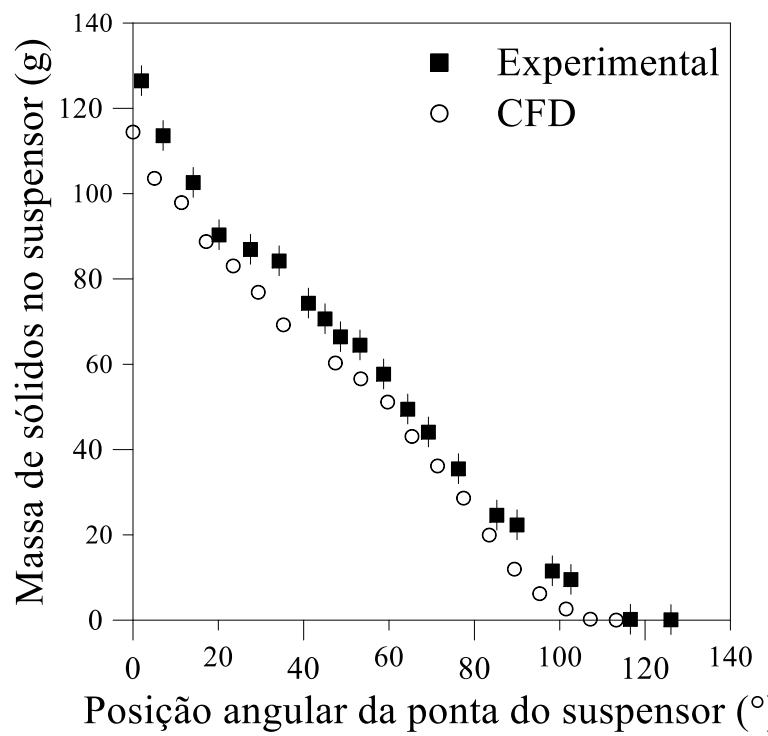

Foi observado nos resultados apresentados pela Figura 6 que a solução numérica apresentou resultados próximos dos experimentais, entretanto o modelo subestimou os valores de holdup e o ponto de última descarga. A média dos desvios entre os resultados simulados e experimental foi em torno de $22 \%$. O ponto experimental da última descarga ocorreu em torno de $125^{\circ}$ enquanto que a simulação previa em torno de $155^{\circ}$. 
Na Figura 7, é apresentado o holdup de sólidos nos suspensores variando com a posição angular para um grau de enchimento do tambor de $3 \%$ para as mesmas partículas. Os resultados simulados e experimentais apresentaram o mesmo comportamento, entretanto o modelo subestimou os pontos de primeira e ultima descarga. A média dos desvios entre os resultados preditos e medidos foi de $24 \%$.

Figura 7 - Comparação entre massas de sólido experimental e simulado para esferas de vidro de 2,56 $\mathrm{mm}$ e carga de sólidos de $3 \%$.

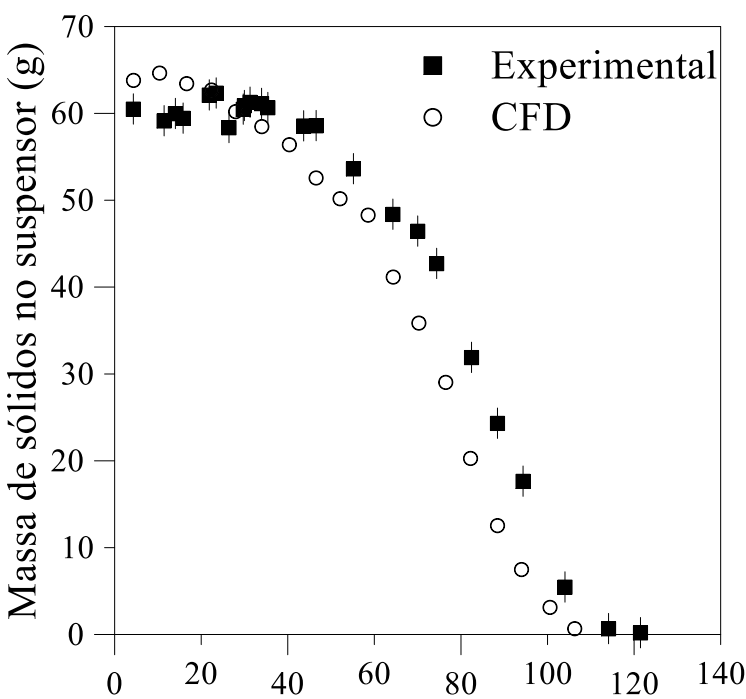

Posição angular da ponta do suspensor $\left({ }^{\circ}\right)$

Investigou-se, também, a aplicação do modelo a outro material (fertilizante SSP com 2,56 mm de diâmetro) em duas condições: sobrecarregamento e subcarregamento (Figuras 8 e 9). Assim como para as esferas de vidro, para o fertilizante houve uma subestimação dos valores de holdup em função da posição angular. A média dos desvios entre os resultados simulados e experimentais foi de 23\%. Para um carregamento de $3 \%$, a carga de sólidos foi também superestimada em posições angulares mais baixas e subestimada em posições angulares mais baixas.
Figura 8 - Comparação entre massas de sólido experimental e simulado para fertilizante SSP de 2,56 $\mathrm{mm}$ e carga de sólidos de $6 \%$.

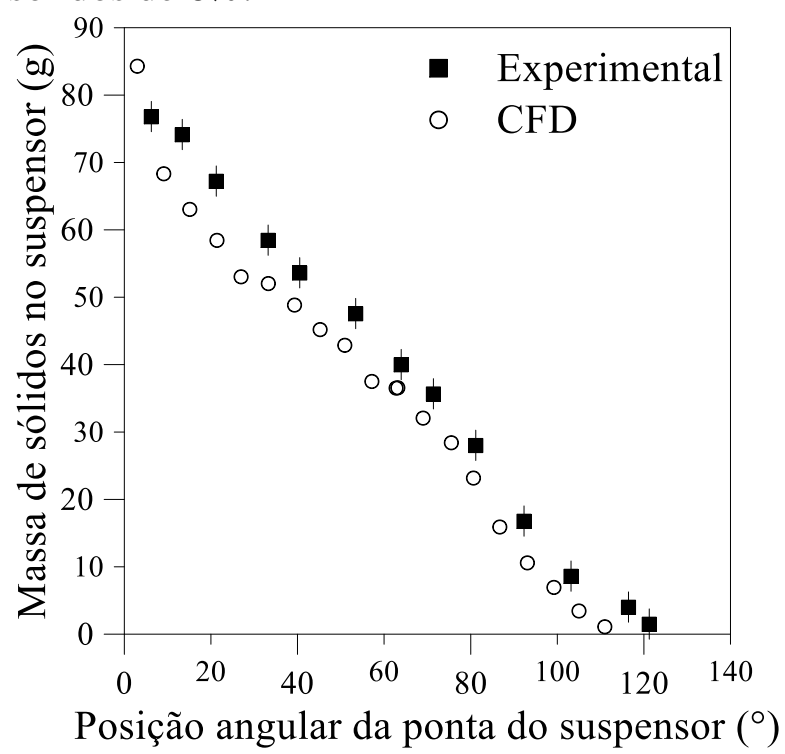

Figura 9 - Comparação entre massas de sólido experimental e simulado para fertilizante SSP de 2,56 $\mathrm{mm}$ e carga de sólidos de $3 \%$.

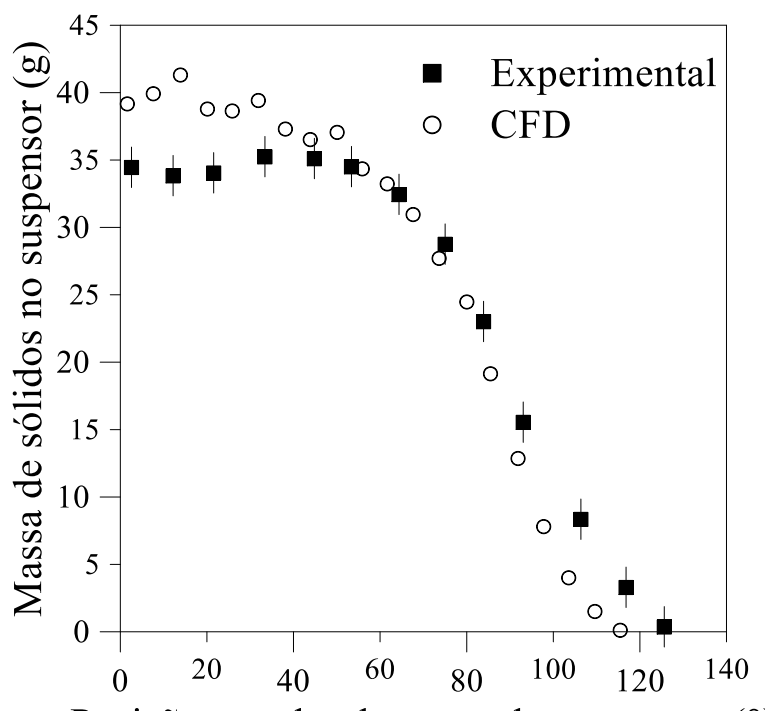

Posição angular da ponta do suspensor $\left(^{\circ}\right)$

Os resultados do estudo mostraram que a abordagem Euleriana foi uma ferramenta capaz de predizer o comportamento da dinâmica de material particulado para diferentes sólidos em tambor 
rotatório com suspensores. Apesar do modelo CFD subestimar os valores da carga de sólidos nos suspensores e o ponto de ultima descarga, esta abordagem permitiu $o$ desenvolvimento de um modelo mais generalizado, com pouca dependência de parâmetros a serem ajustados.

\section{CONCLUSÃO}

Os estudos, numérico e experimental, da dinâmica de material particulado em tambor rotatório mostraram dependência com os ângulos de repouso e diâmetro das partículas estudadas. A carga de sólidos nos suspensores se mostrou independente da velocidade de rotação do tambor.

As simulações foram realizadas utilizando o modelo Euleriano Granular Multifásico acoplado com a teoria cinética do escoamento granular. Uma metodologia foi desenvolvida para estimar a massa de sólidos nos suspensores baseado em resultados de simulação. Em todas as condições simuladas, os resultados em CFD apresentaram um bom ajuste qualitativo em relação aos resultados experimentais, com a média dos desvios entre os resultados simulados e experimentais de $23 \%$. Apesar destas variações, a abordagem Euleriana permitiu o desenvolvimento de um modelo mais generalizado, com baixa dependência de parâmetros a serem ajustados e com um baixo custo computacional comparado a simulações usando DEM.

\section{REFERÊNCIAS}

AJAYI, O.O.; SHEEHAN, M.E. Application of image analysis to determine design loading in flighted rotary dryers. Powder Technol.v.223, p. 123-130, 2012.

GENG, F.; LI, Y.; YUAN, L.; LIU, M.; WANG, X.; YUAN, Z.; YAN, Y.; LUO, D. Experimental study of the space time of flexible filamentous particles in a rotary dryer. Exp. Thermal and Fuid Sci.v. 44, p.708-715, 2013.

GIDASPOW, D., Multiphase Flow and Fluidization, Academic Press, Boston, 1994.

LEE, A. Modelling the solids transport phenomena within flighted rotary dryers, $\mathrm{PhD}$ thesis, James Cook University, 2008.

LUN, C.K.K.; SAVAGE, S.B.; JEFFREY, D.J.; CHEPURNIY N.; Kinetic theories for granular flow: inelastic particles in coquette flow and singly inelastic particles in a general flow field, J. Fluid Mech. v.140, p. 223-256, 1984.

SANTOS, D.A.; PETRI, I.J.; DUARTE, C.R.; BARROZO, M.A.S. Experimetal and CFD study of the hydrodynamic behavior in a rotating drum. Powder Technol.v.250, p. 5262, 2013.

SCHAEFFER, G., Instability in the evolution equations describing incompressible granular flow, J. Differential Equations, vol. 66, p. 19-50, 1987.

SILVÉRIO, B.C.; SANTOS, K.G.; DUARTE, C.R.; BARROZO, M.A.S. Effect of the friction, elastic, and restitution coefficients on the fluid dynamics behavior of a rotary dryer operating with fertilizer.Ind. Eng. Chem. Res.v.53, p.8920-8926, 2014.

WARDJIMAN, A.; LEE, A.; RHODES, M. Behaviour of a curtain of particles falling through a horizontally-flowing gas stream.Powder Technol.v.188, p.110-118, 2008.

\section{AGRADECIMENTOS}

Agradecemos $o$ apoio financeiro concedido pela FAPEMIG, CNPQ e CAPES. 